CAHIERS DE

NARRATOLOGIE

\section{Cahiers de Narratologie}

Analyse et théorie narratives

$36 \mid 2019$

Rhétorique et représentations de la culture mafieuse. Images, rituels, mythes et symboles

\title{
Mafieux ou héros? Mythes et motifs tragiques dans Le Parrain et Le Parrain II de Francis Ford Coppola
}

Rosario Giovanni Scalia

\section{(2) OpenEdition Journals}

Electronic version

URL: http://journals.openedition.org/narratologie/9734

ISSN: $1765-307 X$

Publisher

LIRCES

\section{Electronic reference}

Rosario Giovanni Scalia, « Mafieux ou héros ? Mythes et motifs tragiques dans Le Parrain et Le Parrain I/ de Francis Ford Coppola », Cahiers de Narratologie [Online], 36 | 2019, Online since 20 December 2019, connection on 20 December 2019. URL : http://journals.openedition.org/narratologie/9734

This text was automatically generated on 20 December 2019.

Article L.111-1 du Code de la propriété intellectuelle. 


\title{
Mafieux ou héros? Mythes et motifs tragiques dans Le Parrain et Le Parrain II de Francis Ford Coppola
}

\author{
Rosario Giovanni Scalia
}

\section{Préambule}

Francis Ford Coppola a déclaré à plusieurs reprises que Le Parrain est l'histoire d'un roi qui a trois enfants, et non un film sur la mafia ${ }^{1}$. Dans cette déclaration, la critique a vu une allusion à King Lear de Shakespeare, la grande tragédie sur le pouvoir, plus particulièrement sur une querelle de succession ${ }^{2}$.

À partir des suggestions de Coppola, le parallèle entre la trilogie du Parrain et le genre tragique a connu un certain succès auprès des interprètes de la saga ${ }^{3}$. Cela étant, il nous paraît essentiel de montrer que ce parallèle dépasse les simples analogies thématiques ou les renvois textuels explicites, pour atteindre la structure narrative profonde et les aspects formels du récit, notamment par l'usage récurrent de tópoi éminemment tragiques. L'analyse de ces choix permettra de comprendre les raisons qui ont amené à l'inclusion de la saga de la famille Corleone dans le panthéon des œuvres fondatrices de la mythologie mafieuse.

Tous les thèmes qui jouent un rôle décisif dans Le Parrain s'avèrent être de dérivation tragique. Songeons aux contrastes dialectiques entre liberté et nécessité ou entre clan familial et État, aux archétypes du sacrifice de l'innocent, de la passion incestueuse, de la transmission héréditaire de la souillure, de la violence maternelle à l'égard des enfants. Autant de tópoi qui parlent une langue universelle et préverbale, susceptible d'émouvoir ou d'impressionner le public avant même de frapper son esprit et de solliciter son jugement.

Le caractère fortement pathétique et émotionnel de la narration cinématographique « tragique » de Coppola a donc donné lieu à une réception instinctive et pré-culturelle 
des faits représentés, une réception qui précède et conditionne l'interprétation critique rationnelle.

La caractérisation tragique des personnages de la saga a quant à elle suscité des élans d'admiration sincère, bien qu'inavouables en tant que tels, plutôt que l'exécration et le blâme. Les protagonistes du Parrain sont ainsi entrés de plein droit dans cette galerie de héros tragiques qui, avant même d'appartenir à des récits spécifiques, incarnent des archétypes, des expressions symboliques ancrées dans l'inconscient collectif.

La représentation filmique, en superposant la dimension noble du héros tragique au prototype du chef de clan mafieux, a fini par entourer le monde de la criminalité organisée d'une aura mythique amplifiée par la représentation des Corleone en tant que famille patriarcale traditionnelle, à une époque où ce modèle est fortement remis en cause par les mouvements contestataires, les groupes féministes et la nouvelle culture hippie ${ }^{4}$. Dans le climat culturel des années 1970, le conservatisme et le patriarcat archaïque des Corleone représentent aux yeux du public, un hommage nostalgique et poétique à un monde glorieux en voie de disparition, un Götterdämmerung vécu avec rigueur et dignité et dont le charme contribue de manière décisive au succès du film.

Ce n'est pas un hasard si, dès le début, l'emblème de la trilogie est le personnage le plus conservateur, autoritaire, machiste et homophobe: Don Vito Corleone, le patriarche hiératique rendu immortel par l'interprétation de Marlon Brando ${ }^{5}$. Solidaire avec ce patriarche, le public est fatalement poussé à étendre sa sympathie aux valeurs de la famille mafieuse, arrivant à croire qu'il existe une «bonne mafia» avec laquelle, somme toute, on peut sympathiser.

Le Parrain a joué un rôle fondamental dans la diffusion du mythe de la «bonne mafia » ainsi que d'autres mythes mafieux : son succès planétaire en a fait la principale source d'information sur le phénomène mafieux pour une grande partie de l'opinion. L'imposant Don Vito Corleone, sa voix rauque, son visage boursouflé, sont à ce jour omniprésents, en Sicile, dans toute une série de marchandises répondant aux attentes des touristes venus à la recherche du frisson de la rencontre rapprochée avec cette Arcadie sauvage et primitive peuplée de mafiosi, dont le film de Coppola a offert une représentation suggestive ${ }^{6}$.

Mais le destin commercial et pop du Parrain-Marlon Brando est allé bien au-delà de la simple exploitation marchande et a vécu d'autres vies inattendues : au théâtre, dans la parodie cinématographique, dans la publicité, même dans les dessins animés et les jeux vidéo, donnant à la mafia le visage sévère, mais aussi affable et captivant de Don Vito Corleone ${ }^{7}$.

La bande sonore du film composée par Nino Rota rivalise à son tour avec la renommée internationale de Brando-Corleone. Les thèmes musicaux de Rota ont désormais une valeur iconique et une vie souvent décontextualisée du récit filmique, bien que toujours liée à la sémantique de la mythologie mafieuse. Parmi les Leitmotiven les plus joués dans la Sicile du tourisme, dans les librairies, les boutiques de souvenirs, les restaurants, ils sont le véritable cheval de bataille des musiciens itinérants et le fond sonore obsessionnel du pèlerinage vers les lieux qui ont servi de décor aux longs-métrages de Coppola.

La mafia elle-même s'est emparée des mythes et des icônes du Parrain. Après une lutte acharnée contre Paramount, menée par le chef mafieux Joe Colombo et l'association qu'il a créée à cet effet, la Italian-American Civil Rights League, il y a eu des négociations entre la société de production et la mafia ${ }^{8}$. Cosa Nostra a vite compris que l'image 
romantique et légendaire de la mafia donnée par les auteurs du Parrain était opératoire pour le mythe d'une société de gentlemen, fidèles aux valeurs familiales, vengeurs des opprimés, parcimonieux quant à l'usage de la violence, sages et équitables dans l'administration de la justice, ennemis jurés des affaires «sales» telles que le trafic de drogue, héroïques dans leur posture tragique".

La saga est donc devenue, à bien des égards, une source d'inspiration pour les milieux mafieux, au niveau de la construction mystifiante du mythe de la «bonne mafia » et du développement de certaines stratégies criminelles, mais aussi, plus simplement, lors de l'utilisation de la bande sonore du film comme accompagnement musical aux hommages rendus aux chefs des clans mafieux, sous leurs balcons, lors des processions des statues de saints et de la Vierge ${ }^{10}$.

\section{Liberté et nécessité : une famille maudite?}

D'après Goethe, le sentiment du tragique provient d'un conflit inconciliable entre liberté et nécessité ${ }^{11}$. Inhérent à l'âme humaine, ce sentiment découle de la prise de conscience de la fragilité de la liberté individuelle face aux nécessités de la vie. La tragédie nous apprend que la tentative de l'homme de transformer sa liberté en action n'a pas lieu dans un environnement neutre ou favorable, mais au contact de forces hostiles et fatales, dans un ciel sans dieux. Le sentiment du tragique brise ainsi la confiance optimiste, d'origine humaniste, dans les possibilités de l'homme en tant que faber fortunae suae ou, au sens plus moderne, en tant que self made man.

Dans la tragédie classique, l'un des facteurs qui limite et anéantit le plus souvent la liberté du héros est son héritage familial. Le héros tragique est responsable de ses propres fautes, mais il hérite également de la malédiction qui frappe sa famille en vertu de la faute primordiale (hýbris) commise par le fondateur; une faute qui a produit une modification du kósmos, l'ordre universel du monde.

Or, la transmission de la souillure est sans doute le principal moteur narratif du Parrain. Les deux protagonistes de la saga, Don Vito et son fils Michael, sont des hommes de pouvoir dont la force ne fait que s'accroître. Leur existence est pourtant limitée, dès la naissance, par des contraintes incontournables, héritées de leur passé familial.

Les Corleone sont une famille maudite car contaminée par une hýbris néfaste : au début du XXe siècle, à Corleone, en Sicile, le père de Vito Andolini a osé se rebeller contre le pouvoir absolu de Don Ciccio, qui règne en maître. Puni par la mort et par l'extermination de toute sa famille, le père de Vito n'a pas pour autant épuisé sa culpabilité, qui constitue le lourd héritage de son seul fils survivant, le futur Vito Corleone, dont l'existence sera à jamais marquée par l'acte de hýbris de son père : à l'âge de dix ans, il est déjà seul au monde et, agité par de vagues désirs de revanche, il se dirige vers l'Amérique, à savoir vers l'inconnu, comme tant d'émigrants italiens à la même époque.

La parabole tragique de la nécessité familiale sera répétée à l'identique par Michael, qui devient, malgré lui, l'héritier de son père en tant que chef mafieux. La liberté dont il croit jouir, sa tentative de se délester du fardeau de ses origines en servant son pays pendant la guerre et en fréquentant une jeune fille américaine de bonne famille, l'illusion de pouvoir être différent des siens et même la volonté de son père de le tenir à l'écart des affaires, seront fatalement vouées à l'échec. Quand Michael sera à son tour appelé à sauver la vie de son père blessé dans une embuscade et à le venger, le lien 
étouffant et impérieux de la malédiction familiale prendra le dessus: le sang et l'instinct s'imposent avant même que la raison et le calcul entrent en ligne de compte.

Cette malédiction, comme le veut la tradition tragique, marquera le destin des Corleone de génération en génération, jusqu'à l'extinction de leur sang, qui survient lorsque Kay se fait avorter dans Le Parrain II, puis avec la mort de Michael et de sa fille Mary à la fin du Parrain III.

\section{Vito et Michael Corleone : gangsters ou héros tragiques?}

Le conflit entre liberté et nécessité est d'autant plus frappant qu'un homme riche, puissant et astucieux, donc potentiellement libre de tout conditionnement, en paie le prix. L'élévation du héros, que le théâtre antique soulignait visuellement par l'utilisation des cothurnes portées par les acteurs, sert à souligner la teneur tragique de sa chute.

Vito Corleone possède tous les attributs du héros tragique. Conditionné, comme nous l'avons dit, par un lourd passé, il a fondé son existence sur une éthique simple et irréprochable qui, dans le monde clos de la communauté italo-américaine new-yorkaise où il fait ses premiers pas, lui permet de survivre et le protège de tout excès. Reconnaissant envers ses bienfaiteurs, fidèle et attentionné vis-à-vis de sa femme et des siens, généreux avec ses amis, mais également inflexible face à l'arrogance ou à l'injustice, Vito semble reproduire les schémas mentaux de son père.

Les spectateurs, eux, sont largement familiarisés avec l'éthique élémentaire de Vito: une éthique fondée sur la lex talionis (« œil pour œil, dent pour dent »), déjà présente dans les westerns, à savoir des récits filmiques également caractérisés par des suggestions épiques et tragiques, peuplés de justiciers solitaires et de héros courageux et impitoyables, obligés de vivre dans un monde sauvage et sans lois. Le transfert du système de valeurs du monde chevaleresque du western à l'univers criminel de la mafia a en partie contribué à forger des sentiments de sympathie et d'approbation à l'égard de ce dernier, et à créer une version mafieuse du mythe de la frontière, l'un des mythes fondateurs de l'imaginaire nord-américain.

Comme chacun sait, dans la tragédie classique l'ascension du protagoniste commence par une entreprise héroïque. Voilà donc que, comme ÆEdipe avait libéré Thèbes du Sphinx, Don Vito libère le quartier italien de Brooklyn de son tyran, Don Fanucci.

La scène où Vito tue Don Fanucci est ponctuée d'éléments fortement archétypaux et rituels, qui confèrent au récit une tonalité épique. Après avoir commis le meurtre, Vito rentre chez lui en vainqueur et sa femme, qui l'attend sur le seuil, lui montre un nourrisson, qui n'est autre que le petit Michael ${ }^{12}$. Cette scène permet de saisir la gravité de l'instant de la prise du pouvoir, de la succession dynastique, indissociable de la malédiction familiale destinée à se perpétuer.

Comme tout héros tragique, Vito connait une ascension glorieuse, de sorte que sa chute puisse paraître plus dramatique et impressionnante. Dans la scène d'ouverture du Parrain, il est représenté à l'apogée de son pouvoir : sans bouger de son bureau plongé dans l'obscurité, il est en mesure de déterminer le destin de ses amis et de ses ennemis. Ses succès, de plus en plus retentissants et impressionnants, seront à la mesure de la force de ceux contre qui il se bat: songeons à la séquence où il est question de Woltz, 
riche et puissant producteur hollywoodien, condamné à un réveil macabre dans un lit ensanglanté, la tête coupée de son cheval préféré à ses côtés ${ }^{13}$.

En l'espèce, la brutalité et la vulgarité dont une organisation mafieuse est responsable sont de toute évidence anoblies. Suivant la convention selon laquelle le héros tragique doit avoir des origines aristocratiques, la puissance des Corleone s'inscrit dans la sphère de la royauté, comme en témoigne l'attention que Don Vito accorde aux aspects représentatifs et symboliques de son pouvoir. Dans la scène d'ouverture du film, en vrai monarque, il montre sa répugnance envers l'argent que lui offre Amerigo Bonasera, venu demander son aide, qui s'imagine pouvoir acheter les services du parrain moyennant ses dollars. Tout en méprisant l'argent, Don Vito accorde la plus grande importance au rituel: on s'adresse à lui en utilisant le surnom de "parrain ", on lui rend l'hommage féodal du baisemain, on évoque la supériorité de sa justice par rapport à celle de l'État.

Reste que, comme le veut la tragédie, la toute-puissance n'appartient pas aux humains, et Vito Corleone, qui prétend l'atteindre, commet un acte de hýbris destiné à une sanction inévitable. Ainsi, les coups de revolver de ses rivaux et le temps qui passe lui ôtent la santé puis la vie, mettant fin à sa liberté illimitée. Ayant atteint pour les autres des objectifs qui semblaient irréalisables, il se découvre impuissant au moment de réaliser son propre rêve : libérer sa famille des stigmates du crime.

Dans les dernières scènes où il campe en protagoniste, peu avant sa mort, Don Vito n'est que l'ombre du chef qu'il a été : comme Créon, Richard III, Macbeth ou le fantôme du père de Hamlet, il connaît le déclin de son pouvoir. Errant sans but, frappé à mort, il revoit la séquence des horreurs culminant dans le massacre de Sonny, son aîné, par ses rivaux. Sa voix rauque, qui autrefois semait la terreur chez ses ennemis, est éteinte, sa barbe est négligée, ses cheveux sont ternes : la robe de chambre a remplacé le costume ; dans sa main, pour la première fois, il tient un verre de whisky. Alors qu'il accompagne le corps de son fils au funérarium, il ressent le poids de la solitude et de la vieillesse. À l'instar du vieux Priam désemparé qui, dans l'Iliade, réclame le cadavre sanglant de son fils Hector, Don Corleone veille à enterrer dignement son fils, effaçant de son corps, autant que possible, les signes des blessures infligées par ses bourreaux. C'est un roi qui a perdu sa couronne et qui célèbre dans l'obscurité de son palais le triste rite de l'abdication en faveur de son talentueux successeur, son plus jeune fils Michael. Lors du dernier face-à-face, dans le bureau de Vito, la salle du trône de la famille, lorsque le vieux parrain se lève de son fauteuil, la caméra ne le suit plus et reste centrée sur Michael. La succession a eu lieu.

Mais jusqu'à la fin, Don Vito garde son sang-froid et la maîtrise de soi qui sont l'héritage de sa fierté sicilienne et qu'il a cultivés toute sa vie. Ces caractéristiques correspondent à sa stature tragique : le calme et l'intensité du geste, la force tranquille de la voix et des mots, la détermination, le mépris envers ceux qui sont incapables d'endurance virile et qui pleurent « like a woman », la dignité dans le deuil, la force dont il fait preuve en renonçant à la vengeance pour ouvrir une nouvelle ère de paix entre familles mafieuses.

La représentation de la mort de Don Vito, sous un jour tragique, a lieu dans une scène au lyrisme délicat. Son corps sans vie s'effondre, à l'abri des draps blancs étendus dans le jardin, au souffle d'un vent léger: ce n'est pas à la mort d'un criminel que l'on assiste, mais à un dernier hommage rendu au héros ${ }^{14}$. 
La séquence finale du Parrain, en trois tableaux, développe un symbolisme royal évident: les funérailles somptueuses du vieux monarque permettant à la famille d'étaler son pouvoir et de renforcer ses alliances, sont suivies par le couronnement du nouveau roi, rythmé par le montage alterné du rite baptismal du fils de Connie, la sœur de Michael, et du massacre simultané des ennemis voulu par le jeune parrain. La solennité à la fois sacrée et sinistre ${ }^{15}$ est frappante : Michael devient en même temps parrain de l'enfant que l'on baptise, parrain de la famille mafieuse et "père omnipotent » en tant qu'implacable justicier. On assiste enfin à l'hommage rendu par les vassaux qui s'inclinent et baisent la main du nouveau roi assis sur le trône, le fauteuil du bureau de feu son père.

Les premières images du Parrain II établissent une continuité : on voit justement un baisemain et un gros plan du visage de Michael, dont la moitié seulement est éclairée, ce qui met l'accent sur l'ambiguïté de son pouvoir et sur la division tragique de sa personnalité16. Le nouveau monarque est maintenant prêt, comme l'avait été son père, à gérer son pouvoir et à défaire ses ennemis. A vrai dire, Michael apparaît sous un jour héroïque dès sa première apparition, lors du mariage de Connie, au tout début du Parrain, puisqu'il se distingue dans la foule des invités grâce à son uniforme et aux décorations qui signalent sa vaillance guerrière.

Pour le meilleur et pour le pire, comme tout héros tragique, Michael est un prédestiné ${ }^{17}$. La clé de voûte de sa vie, nous l'avons vu, est l'instant où il se sent appelé à venger son père. Le meurtre du rival Sollozzo constitue en fait son initiation, son entrée dans la famille mafieuse. Comme nous l'avons précisé, les signes de sa prédestination sont toutefois évidents dès sa naissance, et sa condition d'héritier est implicitement signalée par la prédilection de son père, qui le chérit depuis sa plus tendre enfance. Cette prédilection se révélera être une malédiction, une mauvaise fortune.

L'accès au pouvoir de Michael est préparé par un long séjour en Sicile, où il a fui pour se mettre à l'abri d'éventuelles poursuites ou rétorsions, après avoir perpétré la vengeance de son père. Dans le récit, les scènes siciliennes ont tout d'abord une forte valeur symbolique et initiatique.

Avant d'accéder au pouvoir, le héros doit subir une période de purification et de mise à l'épreuve, et doit s'abreuver aux sources d'où ce pouvoir est issu. La Sicile symbolise donc le retour aux origines, le lieu mystique où l'ascension du nouveau parrain peut commencer. Dans une scène emblématique, Michael refuse d'emprunter la voiture pour monter à pied, tel un pèlerin, vers le village perché de Corleone. De même qu'CEdipe se rend chez l'oracle de Delphes pour connaître ses origines, ainsi Michael achève son pèlerinage à la recherche des racines sur lesquelles il va pouvoir se greffer, nouvelle pousse issue d'une plante ancienne.

En ce sens, même sa rencontre avec Apollonia, sa future épouse, dont la vraisemblance sur le plan narratif peut sembler faible, se prête à une lecture symbolique. Comme Béatrice pour Dante, Apollonia est la femme angélique destinée à guider Michael sur le chemin de la pureté, avant de disparaitre à jamais. La chaste étreinte qui suit leur mariage renvoie à l'étreinte entre Michael et ses racines ${ }^{18}$. Peu après, la mort de cette jeune épouse représente de toute évidence l'archétype tragique du sacrifice propitiatoire de l'innocent. De même que le sacrifice de la vierge Iphigénie fut nécessaire pour permettre le départ de la flotte achéenne vers Troie, ainsi la mort d'Apollonia rendra aisé le double voyage, réel et allégorique, de Michael : son retour en Amérique et son accession au pouvoir en tant que nouveau chef de la famille mafieuse. 
Malgré la diversité des personnalités, des époques et des circonstances, l'existence de Michael s'inscrit dans le sillage de celle de son père, $\mathrm{y}$ compris dans la phase de son déclin. Après avoir atteint des sommets plus élevés que Don Vito, Michael va à son tour connaître les limites de l'expérience : la trahison de son frère Fredo et la rébellion de sa femme Kay. Poussée au désespoir par son mari, celle-ci décide de se fait avorter, devenant in fine la réplique de Médée ${ }^{19}$, après avoir lutté pour préserver la liberté des enfants qu'elle a déjà mis au monde et pour tenter d'arrêter le tourbillon de la souillure qui pourrit leur sang. Autant dire un revers fatal pour le nouveau parrain. Le Parrain II s'achève avec la représentation d'un roi puissant mais dramatiquement seul, tourmenté par les fantômes du passé, en proie à ses remords ${ }^{20}$.

\section{La famille et les familles : la logique du ghénos}

Parmi les conflits qui caractérisent la tragédie classique, ceux qui éclatent au sein de la famille occupent une place de choix. En tant que forme primitive de la communauté humaine, la famille permet à la tragédie de saisir les relations interpersonnelles, les tensions et les conflits, dans leur phase embryonnaire, à un stade de spontanéité instinctive et émotionnelle que des formes plus complexes de regroupement social ne peuvent posséder.

La famille est à la fois le lieu et l'emblème des liens ancestraux et indissolubles, inscrits dans l'intimité génétique de chacun de ses membres. Elle est la cause première et inévitable de tout comportement individuel, selon le principe évoqué plus haut, de la responsabilité collective et de la transmission héréditaire des fautes. Enfin, la famille est un ghénos, un clan, un groupe social autonome et autorégulé, qui naît et grandit selon des règles, des contraintes et des lois endogènes qui finissent inévitablement par entrer en conflit avec l'éthique de la pólis, c'est-à-dire avec le système politique et juridique de la communauté élargie dont elle fait partie. Or, le traitement tragique du thème de la famille, avec le corollaire de l'enquête sur les conflits familiaux, sont les moteurs de la saga du Parrain.

Dans le code éthique de Vito et Michael Corleone, les valeurs familiales jouent un rôle fondamental et ont une primauté incontestée. Comme nous l'avons vu, l'existence de Michael est marquée par l'appel ancestral de la loi du sang lors de l'attentat subi par son père. Cet événement éveille son instinct naturel primordial, la phýsis, contre laquelle rien ne peuvent les interdits opposés par la culture et la société (nómos), que Michael a tout de même assimilés pendant sa période d'intégration à la société américaine, en tant qu'étudiant, soldat et fiancé.

C'est précisément la primauté incontestée que la famille et ses règles occupent dans l'horizon mental des Corleone qui détermine, au sein du Parrain, la prépondérance absolue du ghénos par rapport aux valeurs de la pólis. Aucun des membres de la famille Corleone, en effet, ne reconnaît à la pólis cette fonction d'arbitrage et de médiation ou cette stature éthique que le contrat social et le droit sont censés lui conférer. L'État est perçu comme un interlocuteur complaisant qu'il faut corrompre ou bien un ennemi à abattre ou encore une institution à infiltrer.

Le conflit entre ghénos et pólis avait pu émouvoir Michael lorsque, jeune et idéaliste, il avait décidé de s'engager et de se battre pour son pays, en dépit des remarques de son frère Sonny lui rappelant que la famille vient avant la patrie. Ce conflit n'est plus d'actualité pour le boss cynique et désabusé que Michael finit par devenir. 
Plusieurs autres conflits familiaux sont au cœur de la saga des Corleone : le conflit au sein de famille biologique, déchirée entre le modèle patriarcal traditionnel et le modèle nucléaire émergent; le conflit qui oppose la dynamique et les intérêts de la famille biologique à ceux de la famille mafieuse ; le conflit qui oppose, au sein de la famille mafieuse, deux conceptions de la mafia.

Le premier surgit lorsque le modèle familial patriarcal, que Don Vito a transplanté dans le Nouveau Monde en y fondant sa famille, entre en crise sous la pression des nouvelles exigences sociales qui traversent la société américaine. Tandis que Don Vito avait été le chef incontesté de sa famille, capable de plier à son pouvoir la volonté de sa femme et de ses enfants, la situation change radicalement lorsque Michael prend les rênes de la famille.

Pendant son voyage en Sicile, ce dernier a pu connaître les dynamiques profondes de la société patriarcale : grâce à un montage habile, s'alternent à l'écran son immersion dans les rituels de la famille sicilienne traditionnelle (la demande en mariage auprès du père d'Apollonia, sa cour discrète sous les yeux attentifs de la famille de sa fiancée, la chasteté préservée jusqu'à la nuit de noces) et les excès libertins de son frère Sonny, qui épuise les énergies de la famille dans ses visites clandestines à Lucy, sa maîtresse, ainsi que les tromperies et les violences de Carlo, leur beau-frère, à l'encontre de Connie, leur sœur. L'équilibre de la famille sicilienne traditionnelle est donc miné, y compris chez les Corleone, par la dégénérescence pathologique et les dysfonctionnements dus au contexte social américain.

Dans ce tableau affligeant, Michael est le seul à rester fidèle à la loi du père : il ne trompe pas sa femme, il refuse les soirées et les prostituées que son frère Fredo veut lui procurer, il ne cède jamais aux tentations du vice. A la différence de son père, cependant, Michael ne peut éviter d'être confronté aux nouvelles exigences de la famille nucléaire américaine, ni aux revendications que l'émancipation féminine a mises à l'ordre du jour et dont Kay sera la courageuse porte-parole, parvenant au bout du compte à briser le lien conjugal qui la contraignait.

Dans sa dernière conversation avec sa mère, peu avant la mort de celle-ci, Michael est forcé d'admettre que les temps ont profondément changé et qu'il a perdu la maitrise de sa famille, ce qui était impossible dans le monde tel que Madame Corleone l'a connu.

Cette perte est rendue visible dans les dernières scènes du Parrain, où la reconstitution nostalgique d'une fête d'anniversaire du patriarche Don Vito, célébrée par toute la famille, s'oppose à la solitude désespérée à laquelle Michael est forcé, repoussé par sa femme et loin de ses enfants, dont les jouets gisent abandonnés dans le jardin couvert de neige glacée ${ }^{21}$.

L'incapacité de préserver sa famille est la véritable tragédie du jeune parrain, qui, au plus fort de son succès en tant qu'homme de pouvoir, est forcé de subir un échec en tant que mari et père. Le Parrain III ne fera que reprendre le récit de l'échec de Michael pour en montrer les conséquences extrêmes sur son pouvoir et sur son existence.

Le conflit entre la famille biologique et la famille mafieuse impose également des choix tragiques à Michael. Demeuré latent sous Don Vito, qui savait séparer les deux univers (Connie le rappelle à Sonny : «Papa never talked business at the table, and in front of the kids »), ce conflit éclate dans toute sa puissance dévastatrice sous Michael, jusqu'à l'issue fatale de la trahison de Fredo. 
Ayant découvert que son frère l'a trahi, Michael doit décider si sa réaction doit être celle du chef de la famille Corleone ou celle du chef d'une famille mafieuse. Une fois de plus, selon les règles de la tragédie, il va privilégier la responsabilité plutôt que la consanguinité. Il ordonne l'assassinat de Fredo non pas pour éliminer un frère déloyal, mais pour supprimer un élément perturbateur et potentiellement dangereux pour le clan mafieux.

Dans le contexte élargi de la grande famille mafieuse new-yorkaise, les Corleone se trouvent également face à un choix tragique. Don Vito et Michael croient fermement qu'en tant qu'émanation de la famille biologique patriarcale, la famille mafieuse ne peut qu'hériter de sa structure verticale et de ses valeurs, à savoir la loyauté mutuelle et absolue des membres, le respect des hiérarchies, la défense de l'honneur. Leur idéal se heurte à une nouvelle conception entrepreneuriale de la gestion de l'association mafieuse, où la maximisation du profit l'emporte sur la loyauté et l'honneur. Tandis que le chef de la famille Sollozzo, incarnation du nouveau chef d'entreprise, après avoir attenté à plusieurs reprises à la vie de Don Corleone, sollicite un rendez-vous avec Michael croyant pouvoir faire des affaires avec lui, Michael, pour sa part, considère qu'il est impossible de traiter avec l'homme qui a menacé à la fois la vie de son père et l'honneur de sa famille, et ne peut envisager cette rencontre que comme un duel au dernier sang, afin de regagner l'honneur perdu.

Fidèle à une éthique chevaleresque désormais en déclin, Michael finira par rester le seul paladin de l'honneur des Corleone, tandis que l'ancien monde de son père s'effrite. Le sens de l'honneur qui survit en Sicile, a été oublié ou trahi aux Etats-Unis. Même un ancien et fidèle collaborateur des Corleone, Frank Pentangeli, est prêt à coopérer avec la justice afin de se venger de Michael. Ce n'est pas un hasard si Pentangeli changera d'avis après avoir croisé au tribunal son frère, Don Vincenzo, arrivé spécialement de Sicile pour le rappeler à l'honneur familial, qui aurait été fatalement compromis par ses aveux. Don Vincenzo ne s'attardera pas en Amérique : son départ immédiat indique clairement que l'orthodoxie sicilienne condamne sans appel l'hérésie américaine.

\section{Modulations tragiques du récit}

Comme les héros de la tragédie classique, les protagonistes du Parrain évoluent dans un monde à part, à l'écart du commun des mortels, dont ils ne partagent ni l'existence ni la «normalité ». Cet écart est d'abord chronologique, puisque l'histoire représentée à l'écran précède d'une bonne vingtaine d'année l'époque de la sortie du film en salle, mais surtout parce que la narration s'attarde de manière spectaculaire sur le passé mythique des Corleone en lui attribuant une importance démesurée, qu'il est essentiel de saisir si tant est que l'on souhaite parvenir à une compréhension globale de la saga : l'arcanum imperii de la famille, c'est-à-dire l'origine mystérieuse de son pouvoir, doit en effet être recherché loin dans le temps et dans l'espace, à savoir dans la Sicile du début du XXe siècle.

La Sicile semble d'ailleurs se situer à une distance sidérale du présent, tant du point de vue psychologique que du point de vue anthropologique, mais l'héritage de ce monde lointain est toujours pleinement opérationnel au sein de la famille Corleone, présentée comme un groupe social se situant au carrefour de plusieurs langues et cultures. Le scénario nous rappelle constamment cette spécificité, par exemple en insistant sur la polyvalence linguistique de chacun : capables de s'exprimer en anglais, italien, italo- 
américain et en dialecte sicilien, les personnages sont les locuteurs d'un mystérieux idiome qui, à défaut de correspondre à un code connu ou répertorié, sert à certifier l'altérité autant que la solennité de leurs échanges, en produisant un effet d'exotisme destiné à impressionner et charmer les spectateurs.

Entourés d'une aura de mystère dès leurs premiers échanges, les Corleone et leur entourage mènent leurs affaires dans le respect des traditions de la grande histoire. Une scène emblématique, celle le rencontre en prison entre le mafieux Frank Pentangeli et Tom Hagen, l'avocat de la famille Corleone, permet de percevoir la transfiguration du présent grâce à l'évocation de l'histoire ancienne, notamment de l'antiquité romaine. L'objet de la conversation, l'injonction brutale faite au traitre Pentangeli pour qu'il se suicide, est suivie de l'évocation nostalgique par Pentangeli de la grandeur passée de la famille Corleone, qui n'est autre qu'un renvoi à l'histoire de Rome : «the Corleone Family was like the Roman Empire». La réplique de Tom Hagen est à son tour placée dans le contexte prestigieux suggéré par son interlocuteur: l'ordre péremptoire se pare de l'habit élégant de la culture, dès lors que Tom mentionne l'usage romain selon lequel le suicide volontaire du traitre épargnait à sa famille toutes représailles de la part de l'Empereur. Ayant bien compris les instructions recelées dans les mots de Hagen, Frank Pentangeli se suicidera en se saignant dans une baignoire, comme Sénèque et Lucain avant lui, soupçonnés de trahison par Néron, s'étaient volontairement donné la mort. Pour un instant, le public peut oublier la différence abyssale entre les activités criminelles de Pentangeli et les raisons idéales des grands intellectuels romains. C'est du moins l'effet recherché par le récit filmique dès lors qu'il superpose le suicide du truand et la destinée noblement tragique de Sénèque et Lucain. De son côté Michael, aussi impitoyable et puissant que Néron, attendra dans son palais la nouvelle de la mort des conspirateurs, qui une fois de plus est consommée presque simultanément: dans la prison de haute sécurité (Pentangeli), à l'aéroport (Hyman Roth), sur la surface calme du lac Tahoe (Fredo).

Comme dans la tragédie classique, dans Le Parrain aussi les métaphores et les symboles jouent un rôle très important. C'est du moins ce que semble suggérer le réalisateur lorsqu'il introduit dans la séquence de la procession religieuse à Little Italy, un spectacle de marionnettes siciliennes (les célèbres pupi). Il s'agit là d'une allusion au choix d'une réalité complexe où le niveau visible - le jeu des marionnettes sur scène - n'a de sens que par l'existence d'un niveau sous-jacent, invisible au regard, celui où se situent les marionnettistes. Et d'ailleurs, rappelons au passage que le logo du film n'est autre qu'une main stylisée tenant d'invisibles marionnettes, dont on aperçoit juste les fils.

La lecture symbolique est également encouragée par la fréquence de différents tópoi : la présence de rites religieux, l'apparition récurrente d'oranges, la ritualité sacrificielle de certains meurtres.

Le Parrain reprend également de la tragédie l'utilisation paradoxale de l'ironie, de telle sorte que les actions du héros n'aboutissent pas au résultat escompté et mènent à sa perte plutôt qu'à son salut.

En ce sens, on pourrait dire que la saga tout entière est tragiquement ironique, puisque chacun s'emploie à maintenir l'unité familiale, mais au bout du compte Michael actera la dissolution des Corleone.

Le choix des scénaristes et du réalisateur consistant à créer un "long long-métrage " (très long, compte tenu des usages de l'époque ${ }^{22}$ ) correspond également aux critères de la tragédie grecque, où la « longueur " permettait justement de suivre l'évolution d'une 
malédiction familiale au fil des générations, comme c'est le cas par exemple dans les trilogies d'Eschyle consacrées aux familles maudites des Atrides et des Labdacides. Une intention narrative similaire est signalée par la division en cinq actes (au lieu de trois) des tragédies shakespeariennes, ou par la tétralogie wagnérienne. Une structure aussi articulée offre l'opportunité de tisser une toile dense de références et de renvois, organisés autour d'analogies et d'antiphrases qui donnent une forte unité à l'ensemble du récit.

Enfin, la complexité du Parrain est inspirée de la tragédie dans la mesure où la saga des Corleone est à son tour un spectacle total. Depuis ses origines, la tragédie a attribué un rôle essentiel à la musique et au chant, ainsi qu'aux chorégraphies du chœur, aux masques et aux costumes, à la scénographie et même à de rudimentaires effets spéciaux. De ce point de vue, le cinéma, en raison de la variété et de la puissance de ses moyens, est le seul lieu d'art susceptible de recueillir et amplifier l'héritage du spectacle tragique. Les auteurs du Parrain, conscients du potentiel disponible, ont donc construit un spectacle à fort impact visuel, où tous les éléments non verbaux jouent un rôle fondamental et sont déployés afin de maintenir le public dans un état de " séquestration émotionnelle »

\section{Conclusion}

Le caractérisation tragique qu'ont choisie les auteurs du Parrain pour construire leur saga a fortement conditionné le public dans ses jugements sur les événements représentés et a contribué à nourrir le mythe de la mafia, à la fois au niveau ésotérique (la mafia ennoblie aux yeux de ses propres membres) et au niveau exotérique (la mafia "promue » et entourée d'une aura prestigieuse auprès de l'opinion) ${ }^{24}$.

On peut notamment préciser que si la stature tragique conférée aux protagonistes en fait des personnages héroïques, dotés d'un courage et d'une audace extraordinaires, capables d'exploits légendaires, en même temps elle les place forcément au-dessus des lois auxquelles le commun des mortels fait référence. Or, si cette idée nourrit l'orgueil des associés, elle rend aussi la perspective de l'affiliation attrayante pour d'éventuels aspirants. Par ailleurs, en suscitant la peur, le respect ou l'admiration, la prétendue exceptionnalité héroïque de l'expérience mafieuse fait que le public renonce à évaluer les actes criminels représentés sur scène selon les principes de l'éthique courante.

Qui plus est, la fonction d'arbitrage dans la résolution des conflits exercée par le parrain, accrédite l'idée selon laquelle la mafia serait une dispensatrice de justice. Cette justice apparait, certes, primitive, sommaire et impitoyable, et néanmoins elle est d'une efficacité qui fait défaut à l'État, représenté dans ses lenteurs comme étant injuste. Ce n'est pas un hasard si le premier «verdict» de Don Vito est prononcé en faveur d'une jeune femme violée, à qui l'État n'a pas rendu justice ${ }^{25}$.

La défense acharnée, quoique vaine, des valeurs familiales mise en place par Don Vito et son fils Michael suggère qu'il existe une "mafia traditionnelle », fidèle aux valeurs du ghénos qui, malgré la dégénérescence de la société actuelle, exalte l'unité du foyer, la fidélité conjugale, le respect des hiérarchies et des aînés. Paladins de ce modèle familial, les Corleone incarnent un paradoxe servi au public sans ménagement: truands, les voilà promus à censores morum, détracteurs attitrés d'une modernité qui a oublié et trahi la tradition. 
L'État corrompu et inefficace et ceux qui l'incarnent ou le défendent ne sont que des hypocrites méprisables aux yeux du public : sensibles à la flatterie de l'argent, proies faciles de toute sorte de chantages, bref, paladins d'une moralité douteuse, ils ne font pas le poids face à Don Vito et Michael qui, eux, incarnent le respect des valeurs.

Insistant sur la question de l'honneur, les auteurs mettent en avant le lien entre la mafia, l'éthique chevaleresque et les codes de comportement les plus anciens de la tradition occidentale, auxquels remonte le principe "honneur ou mort», ce qui finit par suggérer l'assimilation des chefs mafieux, les soi-disant hommes d'honneur, aux héros de l'antiquité ou aux chevaliers sans peur et sans reproche de la chanson de geste. Sans oublier que l'utilisation d'un langage essentiellement allégorique, la place consacrée à des aspects symboliques et rituels semblables à ceux qui caractérisent les sectes et les cultes mystiques, ne font que renforcer le charme et le mystère de l'organisation criminelle.

Par ailleurs, la lutte acharnée que les Corleone mènent contre les familles mafieuses rivales, qui trahissent les valeurs du sang et de l'honneur au nom de la logique du profit, accrédite l'existence d'une "bonne mafia », qui ne gère que des trafics véniels tels que le jeu, la contrebande ou le proxénétisme. Le refus catégorique opposé par Don Vito, au péril de sa vie, à l'exploitation du narcotrafic par les familles mafieuses nordaméricaines, accentue dans la fiction le côté vertueux de cette «bonne mafia » animée de scrupules éthiques et armée d'une conscience morale dont, dans la vie réelle, on n'a jamais vu la trace et, dans la chronique judiciaire, le témoignage.

Les scènes siciliennes du film et les flash-backs consacrés aux origines des Corleone conduisent à croire que les "bons mafieux » sont issus des classes populaires et que la «bonne mafia » est née jadis en réaction aux abus de pouvoir subis par le bon peuple à cause d'oppresseurs tels Don Ciccio et Don Fanucci, que Don Vito se chargera d'éliminer. Cette vision édifiante et idyllique est largement démentie par les travaux des historiens, qui ont bien montré que le phénomène mafieux est le fait des classes dirigeantes ${ }^{26}$.

Les prototypes du chef mafieux, de la famille mafieuse, du comportement mafieux construits dans le long-métrage ont contribué à la construction d'une image mythique fictive de la mafia qui a eu un impact certain sur la véritable mafia elle-même, dont on sait qu'elle a notamment adopté des postures non-verbales propres du langage corporel mafieux scénarisés par Coppola.

Pour mesurer l'impact du Parrain on pourrait emprunter à Jean Baudrillard la notion de " simulacre ", à savoir une copie à l'identique d'un objet original n'ayant jamais existé ou, si l'on préfère, un «mythe » qui, dépourvu de réalité, conditionne pourtant la réalité et la dépasse de par sa capacité à être reconnu auprès d'une communauté, devenant ainsi une hyper-réalité27. Reste que sans la caractérisation tragique du Parrain dont nous avons explicité les lignes essentielles, un tel transfert de la fiction à la réalité n'aurait sans doute jamais pu avoir lieu. 


\section{BIBLIOGRAPHY}

Boyle, Nicholas (2010), « Goethe's Theory of Tragedy », The Modern Language Review, 105.4, October, pp. 1072-1086.

Citron, Marcia J. (2004), « Operatic Style and Structure in Coppola's Godfather Trilogy », The Musical Quarterly, 87.3, Autumn, 423-467.

Coppola, Francis Ford (2016). The Godfather Notebook, New York, Regan Arts.

Cristaldi, Cirino (2016), La mafia e i suoi stereotipi televisivi, Barrafranca (EN), Bonfirraro.

Dainotto, Roberto M. (2015), The Mafia : a Cultural History. London, Reaktion Books.

Eckermann, Johann Peter (1969), Colloqui con Goethe, 2 voll. Torino, Utet.

Goleman, Daniel (1996), Emotional Intelligence, London, Bloomsbury.

Lebo, Harlan (1997), The Godfather legacy, New York, Fireside.

Lewis, Jon (2010), The Godfather. London, Palgrave Macmillan.

Nicaso, Antonio (2016), Mafia, Torino, Bollati Boringhieri.

Nicaso, Antonio et Marcel Danesi (2013), Made men. Mafia Culture and the Power of Symbols, Rituals and Myth, Lanham (MD) - Plymouth (UK), Rowman \& Littlefield.

Nicaso, Antonio et Scalia, Rosario Giovanni (2019), La mafia e il mito, in A. Giorgi (éd.), Mai più nell'ombra, Milano, Franco Angeli, pp. 187-213.

Phillips, Gene P. et Rodney Hill, éd. (2004), Francis Ford Coppola. Interviews. Jackson, University Press of Mississippi.

Poon, Phoebe (2006), « The Tragedy of Michael Corleone in The Godfather Part III », Literature/Film Quarterly, 34.1, pp. 64-70.

Schumacher, Michael (1999), Francis Ford Coppola. A Film Maker's Life. New York, Three Rivers Press.

Schwanebeck, Wieland (2010), « From Shakespeare's Kings to Scorsese's Kingpins: Contemporary Mob Movies and the Genre of Tragedy », aspeers, 3, pp. 69-90.

Solomon, Jon (2015), “ "Oedipus... The Structure of Funny" : Allusions to Greek Tragedy in Contemporary Cinema », Illinois Classical Studies, 40.2, Fall, pp. 373-389.

Szigeti, Balázs (2009), « The Dialects of Sin in Shakespeare's Macbeth and Francis Ford Coppola's The Godfather Trilogy ", The AnaChronisT, 14, pp. 24-46.

Szondi, Peter (2003), Essai sur le tragique, traduit par Jean-Louis Besson, Oberhaubergen, Circé.

\section{NOTES}

1. A propos du roman de Mario Puzo, duquel il s'est inspiré pour son film, Coppola a précisé : « Upon that second reading, much of the book fell away in my mind, revealing a story that was a metaphor for American Capitalism in the tale of a great king with three sons: the oldest was given his passion and aggressiveness ; the second his sweet nature and childlike qualities; and the third, his intelligence, cunning and coldness ", Coppola 2016, pp. xx-xxiv. Par ailleurs, aux 
critiques qui lui reprochent d'avoir donné une image romantique de la mafia américaine, il répond : «It is a mistake to think I was making a film about the Mafia. Godfather I is a romance about a king with three sons. It is a film about power " (in Phillips - Hill 2004, p. 19). Coppola ajoute des détails dans un autre entretien : «But, as I've said, this movie was never about a Mafia family. I think it was about a classic noble family. It could be about kings in ancient Greece or in the Middle Ages. It could just as well be about the Kennedys or the Rothschilds, about a dynasty that transcends even one's obligations to one's country. It is about power and the succession of power » (in Lebo 1997, p. 216). Les déclarations de Coppola semblent exagérées, car à l'origine du Parrain il y a un sujet de Mario Puzo, dont le titre original était bel et bien Mafia. A cause des pressions exercées par le chef mafieux Joe Colombo par l'intermédiaire de son Italian-American Civil Rights League les mots «mafia » et « Cosa Nostra " ne seront pas utilisés dans le scénario (cf. Lebo 1997).

2. Cf. Dainotto 2015.

3. Cf. Szigeti 2009, Poon 2006 et Citron 2004. Sur la relation entre mafia et tragédie au cinéma, cf. Schwanebeck 2010.

4. Coppola dit avoir été obligé d'antidater l'histoire aux années quarante (le récit de Puzo se situe dans années 1970), car un contraste trop marqué aurait opposé la culture patriarcale des Corleone à celle des hippies (in Phillips - Hill 2004, p. 172). A ce propos, l'un des scénaristes du film, Robert Towne, ajoute : «In the seventies, when we felt families were disintegrating, and our national family, led by the family in the White House, was full of backstabbing, here was this role model of a family who stuck together, who'd die for one another. The real appeal of the movie was showing family ties in a setting of power. It was really kind of reactionary in that sense - a perverse expression of a desirable and lost cultural tradition, filling people with longing for a family like that, a father who not only knew what was best but, if a guy was giving you a hard time, could have someone kill him » (in Phillips - Hill 2004, pp. 181-182).

5. Lors du choix des acteurs, Coppola et son directeur du casting Fred Roos étaient bien conscients des aspects charismatiques du personnage de Don Vito Corleone : « We couldn't find a real Italian actor with the charisma he needed to have. There's a lot of talk about the Don in this movie; he permeates the movie even when he's not onscreen... ( (in Phillips - Hill 2004, p. 173). En revenant sur le casting, Coppola ajoute : «It became apparent that the role called for an actor of such magnetism, such charisma, just walking into a room had to be an event » (Id., p. 20).

6. Cf. Cristaldi 2016, pp. 42-45.

7. A ce propos, voir l'article d'Andrea Meccia, dans ce $\mathrm{n}^{\circ} 36$ des Cahiers de Narratologie.

8. Au mois de juillet 1970 , l'association créée par Joe Colombo organise un rassemblement au Madison Square Garden où une somme d'environ $600.000 \$$ est recueillie afin de bloquer le tournage $\mathrm{du}$ film. Cette mobilisation officielle a été suivie de menaces téléphoniques, fausses alertes à la bombe, etc. (cf. Lebo 1997, pp. 41-44). Dans un entretien paru dans Playboy en 1975 Coppola déclare que Puzo lui avait suggéré d'éviter tout contact avec les mafieux, mais qu'il savait qu'il avait eu plusieurs rencontres entre Al Ruddy, producteur de Paramount, et des représentants de la mafia (cf. Phillips - Hill 2004, pp. 29-30). Sur les rencontres et les accords entre la société de production et Cosa Nostra, voir Lebo 1997, pp. 90-93.

9. À propos du rapport entre la mafia et Le Parrain cfr. Dainotto 2015, où il est question de la satisfaction que montrait Leoluca Bagarella, boss de la mafia sicilienne, lorsqu'on l'appelait « Le Parrain", ainsi que de son admiration pour la bande sonore du film, qu'il a choisie comme musique de son mariage.

10. A Paternò, en 2015, lors de la fête de la Sainte-Barbe, l'un des gros cierges en bois qui suivaient la statue portée en procession s'est arrêté sous le balcon d'un chef mafieux local pour lui rendre hommage, tandis que la fanfare jouait le thème du Parrain (cf. Corriere della Sera, 3 décembre 2015). Ce même thème musical a été utilisé lors de funérailles de mafieux (cf. notamment l'enquête de Giovanni Bianconi, parue dans Corriere della sera le 17 juillet 2018, à 
propos des funérailles du boss Vittorio Casamonica ainsi que l'article d'Andrea Meccia dans ce $\mathrm{n}^{\circ}$ 36 des Cahiers de Narratologie).

11. Cf. J. W. Von Goethe, Gedenkausgabe der Werke, Briefe und Gespräche, éd. Ernst Beutler, XXIV vols., Zürich, Artemis, 1948-1954, XXIII, pp. 348-349. Cf. aussi Szondi 2003 et Boyle 2010.

12. Robert Towne définit cette scène " one of the most chilling, heartrending sequences in movies ", puisque " at the very instant in his life when he moves to save the lives of his children, he damns himself and, as we'll see, them » (in Phillips - Hill 2004, p. 182).

13. Il s'agit de la longue séquence où Tom Hagen, émissaire du parrain, se rend en Californie pour demander à Woltz de « choisir » Johnny Fontane, protégé du parrain, comme protagoniste de son prochain film. L'entrée de Hagen, minuscule silhouette, dans les gigantesques studios hollywoodiens du producteur, filmée en plan large, sert à montrer la puissance de Woltz. Mais cet étalage de grandeur sert surtout à impressionner le public en vue de la victoire du parrain, capable de prendre le dessus même sur le plus fort des ennemis. Dans la dernière scène de la séquence, la caméra s'éloigne de la villa de Woltz au moment où on entend un cri effrayant : après avoir frappé celui qui a osé s'opposer au parrain, ses «soldats » se replient, laissant derrière eux le sang et le désespoir de son adversaire.

14. À propos de la scène qui précède la mort du parrain, où celui-ci joue avec son petit-fils, Coppola a commenté l'idée de Marlon Brando de gonfler ses joues pour amuser le petit, en ces termes : « Of course! The Godfather dies as a monster ! ». Mais l'analyse filmique de la scène, loin de détecter une telle monstruosité, semble plutôt suggérer la hiératique dignité du protagoniste (cf. Phillips - Hill 2004).

15. Sur le montage et la complexité technique de cette scène, cf. Id., pp. 179-180.

16. L'alternance lumière/obscurité est toujours significative: les évènements qui ont lieu en pleine lumière sont le résultat des décisions prises dans l'obscurité du bureau du parrain (cf. Id., pp. 175-176).

17. Coppola le dit lui-même : «Michael Corleone is doomed » (in Id., p. 27).

18. Le prénom choisi pour la jeune fille n'est pas le fruit du hasard. Selon la tradition, Sainte Apolline (ou Apollonie), vierge et martyre, se jeta dans le feu pour ne pas abjurer sa foi. De la même manière, la femme de Michael, tuée dans l'explosion de sa voiture, gardera à jamais sa pureté, loin des risques de la corruption américaine (cf. Solomon 2015, pp. 376-377).

19. Le rapprochement entre Kay et Médée est implicitement confirmé par Coppola, qui définit l'avortement volontaire de Kay «a symbolic statement of power » (in Phillips - Hill 2004, p. 32). Rappelons au passage que Médée tue ses fils pour se venger de son mari Jason et affirmer son pouvoir sur lui.

20. Le Parrain II aurait dû être le volet ultime de la saga des Corleone. Le Parrain III est en fait une production tardive, réalisée suite aux pressions exercées par Paramount sur Puzo et Coppola (cf. Schumacher 1999, pp. 411-434).

21. La scène en question est celle du retour de Michael, après longtemps et en plein hiver, dans sa villa du lac Tahoe. Dans une des chambres, Michael aperçoit Kay, assise à la machine à coudre (Coppola a dit s'être inspiré de Pénélope, cf. Solomon 2015, p. 376).

22. À propos du soutien de Paramount à Coppola en vue de la réalisation d'un si long film, cf. Phillips - Hill 2004, pp. 180-181.

23. Sur la notion de "séquestration émotionnelle " (emotional hijacking), qui indique un état psychologique transitoire où l'on est possédé par ses propres émotions au point que l'approche rationnelle à la réalité est affaiblie, voire impossible, cf. Goleman 1996, I.2.

24. Sur les mythes mafieux ésotériques et exotériques cf. Nicaso - Scalia 2019, pp. 197-209.

25. Cette image délétère de la justice se trouve aussi dans d'autres textes fondateurs de la mythologie mafieuse. Songeons notamment à I Beati Paoli et à la légende des Vendicosi (les vengeurs), où se mélangent l'archétype de l'ombre et celui du chevalier noir, le justicier implacable (personnifié par Robin des Bois, par exemple). Beati Paoli est le nom d'une société 
sécrète dont l'histoire fut redécouverte lors de la publication du roman homonyme de William Galt (pseudonyme de Luigi Natoli) en 1909. Les Vendicosi sont une société secrète de justiciers dont les traces se trouvent déjà dans des manuscrits du XII ${ }^{\mathrm{e}}$ siècle. Beati Paoli et Vendicosi sont rangés par les mafieux au nombre de leurs ancêtres (voir à ce propos Nicaso - Danesi 2013).

26. Cf. le bilan historiographique dressé in Nicaso 2016.

27. Cf. Nicaso et Danesi 2013.

\section{ABSTRACTS}

Le Parrain et Le Parrain II de Francis Ford Coppola constituent l'une des principales sources d'information sur la mafia dont disposent les spectateurs du monde entier. La caractérisation tragique attribuée aux personnages de la saga des Corleone et aux événements dont ils sont les protagonistes ont contribué à rendre iconique et légendaire le stéréotype du chef mafieux et à consolider le mythe d'une «bonne mafia» exprimant les valeurs traditionnelles de la famille patriarcale et dotée d'un sens aigu de la justice et de l'honneur. Cet article souhaite expliciter les principaux motifs tragiques de la saga et les relier aux stéréotypes sur la mafia largement répandus auprès du public. On soulignera notamment que Cosa Nostra elle-même a fini par s'approprier l'image de la mafia proposée dans les films de Coppola, en la considérant opératoire pour la mythologie ésotérique et exotérique de l'organisation.

Il Padrino e Il Padrino II di Francis Ford Coppola costituiscono tutt'oggi una delle principali fonti d'informazione sulla mafia per il pubblico mondiale. La caratterizzazione tragica conferita ai personaggi della saga dei Corleone e alle vicende di cui sono protagonisti hanno contribuito a rendere iconica e leggendaria la figura del capomafia e a consolidare il mito di una « mafia buona ", espressione dei valori tradizionali della famiglia patriarcale e dotata di un acuto senso della giustizia e dell'onore. Questo saggio si ripropone di esplicitarne i principali motivi tragici e di metterli in relazione con gli stereotipi sulla mafia diffusi presso gli spettatori. In particolare si sottolineerà come la stessa Cosa Nostra abbia finito per impadronirsi dell'immagine della mafia proposta dai film di Coppola, considerandola funzionale alla mitologia esoterica ed essoterica dell'organizzazione.

\section{INDEX}

Chronological index: XXe siècle

Mots-clés: mythe mafieux, Cosa Nostra, représentation de la mafia, Le Parrain, Le Parrain II

Geographical index: États-Unis, Italie

\section{AUTHOR}

\section{ROSARIO GIOVANNI SCALIA}

Rosario Giovanni Scalia est professeur de latin et grec dans les lycées. En 2002, il a obtenu un doctorat en philosophie et histoire des idées à l'Université de Catane et, depuis 2016, il est également professeur associé de latin dans la même université. En 2018-2019, il a été boursier 
postdoctoral à la faculté de philosophie de l'Universidade Federal de São Paulo. Depuis 2016, il poursuit également des études en vue d'un deuxième doctorat, en études littéraires, à Middlebury College. Parmi ses publications récentes, on peut citer : « Mafia e mito : un rapporto dialettico ", in The representation of the Mediterranean World by Insiders and Outsiders, A. C. Vitti \& A. J. Tamburri (dir.), New York, Bordighera Press 2018 ; « La mafia e il mito » (avec Antonio Nicaso), in Mai più nell'ombra, A.Giorgi (dir.), Milan, FrancoAngeli 2019. 